\title{
Incorporation and effects of impurities in different growth zones within basic ammonothermal GaN
}

\author{
Sakari Sintonen ${ }^{\mathrm{a}, *}$, Pyry Kivisaari ${ }^{\mathrm{b}, \mathrm{c}}$, Siddha Pimputkar ${ }^{\mathrm{d}}$, Sami Suihkonen ${ }^{\mathrm{e}}$, Tobias Schulz ${ }^{\mathrm{a}}$, James S. Speck ${ }^{\mathrm{d}}$, Shuji \\ Nakamura $^{\mathrm{d}}$ \\ ${ }^{a}$ Leibniz Institute for Crystal Growth, 12489 Berlin, Germany \\ ${ }^{b}$ Engineered Nanosystems group, Aalto University, 00076 Aalto, Finland \\ ${ }^{c}$ Division of Solid State Physics, Lund University, 22100 Lund, Sweden \\ ${ }^{d}$ Materials Department, Solid State Lighting E Energy Electronics Center, University of California, Santa Barbara, CA 93106-5055 \\ ${ }^{e}$ Department of Micro and Nanosciences, Aalto University, P.O. Box 13500, FI-00076 Aalto, Finland
}

\begin{abstract}
The ammonothermal method is one of the most promising candidates for large-scale bulk GaN growth due to its scalability and high crystalline quality. However, emphasis needs to be put on understanding the incorporation and effects of impurities during growth. This article discusses how impurities are incorporated in different growth zones in basic ammonothermal GaN, and how they affect the structural, electrical and optical properties of the grown crystal. The influence of growth time on the impurity incorporation is also studied. We measure the oxygen, silicon, and carbon impurity concentrations using secondary ion mass spectrometry, and measure their effect on the lattice constant by high resolution x-ray diffraction (HR-XRD). We determine the resulting free carrier concentration by spatially resolved Fourier transform infrared spectroscopy and study the optical properties by spatially resolved low-temperature photoluminescence. We find that oxygen is incorporated preferentially in different growth regions and its incorporation efficiency depends on the growth direction. The oxygen concentration varies from $6.3 \times 10^{20} \mathrm{~cm}^{-3}$ for growth on the $\{11 \overline{2} 2\}$ planes to $2.2 \times 10^{19} \mathrm{~cm}^{-3}$ for growth on the (0001) planes, while silicon and carbon concentration variation is negligible. This results in a large variation in impurity concentration over a small length scale, which causes significant differences in the strain within the boule, as determined by HR-XRD on selected areas. The impurity concentration variation induces large differences in the free carrier concentration, and directly affects the photoluminescence intensity.
\end{abstract}

Keywords: A1. Impurities, A2. Ammonothermal crystal growth, A2. Single crystal growth, B1. Bulk GaN, B1. Nitrides

2010 MSC: 00-01, 99-00

\section{Introduction}

Solid-state lighting based on III-Nitride (III-N) semiconductors is currently transforming the industry of general lighting with its high efficiency and rapidly reducing cost [1, 2]. The very promising initial developments in using native GaN substrates open up a wide range of new possibilities to engineer efficient and functional GaN devices. As one example, Hurni et al. have recently demonstrated groundbreaking LED performance with LED heterostructures grown directly on GaN substrates [3]. On the electronics front, access to cheap high-quality bulk GaN substrates is expected to make GaN a serious alternative for power electronics [4]. The ability to grow devices directly on GaN would also most likely provide enormous possibilities to engineer next-generation optoelectronics and photonics based on novel nanostructure

\footnotetext{
*Corresponding author

Email address: sakari.sintonen@ikz-berlin.de (Sakari Sintonen)
}

active regions [5, 6, 7, 8. To fully realize the potential benefits of epitaxial growth on bulk GaN, the GaN substrate fabrication still needs considerable developments in order to provide large and cost-effective bulk crystals with high structural quality, controlled electrical properties and high transparency for optical devices.

Today's commercially available native GaN substrates are mostly based on hydride vapor-phase epitaxy (HVPE), which allows higher bulk growth rates than the very similar metalorganic chemical vapor deposition (MOCVD) method typically used in epitaxial III-N device fabrication. Nevertheless, fundamentally different methods such as Na flux and the ammonothermal method have also gained large interest in the recent years [9]. In particular, the ammonothermal method allows scalable production of highquality material due to the possibility of scaling the autoclaves to accommodate multiple and larger seed crystals. Being one of the most promising emerging techniques to grow bulk GaN crystals, the ammonothermal method has been studied and developed substantially in the last few 
years [10, 11, 12, 13, 14]. 15, 16, several issues limit the maximum crystal size obtainable with the ammonothermal method. In particular, ammonothermal growth typically incorporates high concentrations of impurities in the crystal. The impurities induce lattice strain, which can be detrimental to device epitaxy, lower the substrate transparency and limit the control of electrical properties. Sudden and large enough changes in impurity concentration may ultimately induce enough strain to cause cracking of the substrate. The spatial distribution and effects of different impurities are not yet well known, and to improve the growth and impurity control, studies are needed that map the density and electrical activity of the most important impurities at different stages and different zones of the growing boule.

In this article, we study the distribution of impurities in different growth zones in ammonothermal GaN and determine their effects on the structural, electrical and optical properties. The oxygen, silicon, and carbon impurity concentrations in different growth regions are measured by secondary ion mass spectrometry (SIMS), and their effect on the lattice constants is determined by high resolution x-ray diffraction (HR-XRD). To study the electrical effects of the impurities, we perform position-dependent Fourier transform infrared (FTIR) reflection measurements to obtain the free carrier concentration. Position-dependent low-temperature photoluminescence (PL) measurements are utilized to investigate the band edge and defect luminescence. To determine whether the impurity concentration changes during growth, we perform an artificial interrupted growth study by slicing one ammonothermally grown GaN boule into pieces and removing material by polishing to reveal surfaces of interest. This enables characterizing the different growth zones at several growth times using conventional surface preparation and characterization methods.

\section{Theory \& experiments}

\subsection{Crystal growth $\&$ sample preparation}

The studied GaN boule was grown on a $346 \mu \mathrm{m}$ thick c-plane HVPE GaN seed crystal sourced from Mitsubishi Chemical Corporation, using the basic ammonothermal method with $\mathrm{Na}$ as the mineralizer. An etchback step of six hours was performed in the beginning with temperatures of $575{ }^{\circ} \mathrm{C}$ and $545{ }^{\circ} \mathrm{C}$ in the top and the bottom of the autoclave. The following growth step lasted 28 days with temperatures of $600{ }^{\circ} \mathrm{C}$ and $560{ }^{\circ} \mathrm{C}$ in the bottom and top of the autoclave, yielding a total thickness of approximately $1360 \mu \mathrm{m}$. The peak pressure was roughly 260 MPa. Specifically for the ammonothermal growth of the samples studied in this paper, a high-purity silver capsule with non-hermetic seals on both ends was used to improve the purity of the growth environment. The non-hermetic seals enables pressure balancing inside and outside the capsule while also ensuring minimal mass transfer into and out from the capsule, yielding an ultra-high-purity growth environment 13. Further details on the autoclaves, filling stations, and other important aspects of the growth procedure are also given in Ref. 13 .

In order to obtain surfaces representative of different growth regions and growth times, the as-grown boule was cut along the m-planeinto two pieces and each piece was thinned by diamond polishing plates using descending grit sizes. After each change of grit size, at least three times as much material was polished away as the previous grit size in order to remove the subsurface damage caused by the previous grit (e.g., at least $18 \mu \mathrm{m}$ was removed after a grit size of $6 \mu \mathrm{m})$. The surfaces of both samples were finished with $0.5-6 \mu \mathrm{m}$ diamond particle sizes. Figure 1 shows (a) top-view and (b) cross-sectional reflected light images of one of the studied samples. The crystal is composed of a seed region and several wing regions, which are regions of lateral expansion in a- and m-directions. The seed region and one a-plane wing region are annotated in figure 1. The seed region consists of GaN grown in the Gapolar $(+\mathrm{c})$ and N-polar (-c) directions, while the a-plane wing region contains two faces grown in the m-directions and one face grown in the a-direction. Although lateral expansion occurs in both the a- and the m-directions, the sizes of the m-plane wing regions are negligible due to the extremely slow growth rate of the m-planes [17. We will therefore limit all following discussion of wing regions to a-plane wing regions only. The unstable a-plane growth is sometimes converted to a faceted growth, and the stable m-plane growth is sometimes partially converted to growth on inclined $\{10 \overline{1} 1\}$ planes. The m-planes of the studied sample remained perpendicular to the c-planes, but the a-planes converted to inclined planes at an unknown time during growth of the boule. As seen in the cross-section in figure 1 (b), the facet inclination was determined to be $\approx 58.2^{\circ}$ with respect to the - c-plane. This value is very close to $58.4^{\circ}$, the inclination angle of the $\{11 \overline{2} 2\}$ planes, indicating growth proceeded in the [11 $\overline{2} 2]$-direction.

\section{[Figure 1 about here.]}

An illustration of the cross section of the grown crystal is shown in figure 2 (a), and a diagram of the two cut and thinned samples, Sample 1 and Sample 2, is shown in figure 2 (b). The crystal dimensions were determined from optical images and the Ga-polar to N-polar thickness ratio of $t_{\mathrm{Ga}}: t_{\mathrm{N}} \approx 1: 5$ was measured from a cross-sectional differential interference contrast microscope (DICM) image. Figure 2 is otherwise drawn to scale, but the dimensions of the seed crystal are understated to emphasize the lateral growth.

\section{[Figure 2 about here.]}

As seen in figure 2 (a), due to different growth rates, the thicknesses of the Ga-polar and N-polar regions diminish toward the wing edges, while the wing regions grow 
thicker. The position of the vertical/lateral growth interface of a given surface is therefore determined by its distance to the seed crystal. The seed region is often described as the region directly above or below the seed crystal, and the wing region as the rest, i.e. the expansion area. This is a slightly misleading convention, because the expansion area does not necessarily correspond to exclusive lateral growth. In this study, the term seed area is used to denote Ga-polar and N-polar growth, while the lateral growth regions will be referred to as the wing regions as shown in figure 2 (a).

The studied surfaces shown in figure 2 (b) were obtained by thinning the as-grown crystal. The surfaces were chosen to provide a reference point on Ga-polar growth and its associated wing region, and two reference points on N-polar growth and their corresponding wing regions. In order to account for a potentially time dependent ambient impurity concentration, comparisons between different growth regions should be made under identical growth conditions. Since the growth rates of ammonothermal Ga, N- , m-, and a-plane growths are markedly different [17, regions grown at equal times should be compared, rather than regions equidistant from the seed crystal. For this purpose, the distances of the surfaces in figure 2 (b) were converted to growth times, and dotted lines were drawn in figure 2 to represent growth evolution at 7 day intervals, thereby assuming a constant average growth rate throughout growth.

The top surface of Sample 1 consists of a maximum of $100 \mu \mathrm{m}$ of GaN grown above the seed crystal in the Gadirection. This distance corresponds to a growth time of 15 days and the surface will hereafter be called Ga15Days. The seed area on the backside of Sample 1 is composed of $750 \mu \mathrm{m}$ GaN grown in the -c direction, corresponding to 27 days of growth. Sample 2 was cut similarly, but with both surfaces grown in the N-direction. The top surface of Sample 2 corresponds to 14 days of growth, while the backside of Sample 2 was cut identically to Sample 2 in order to use it as a control surface. The surfaces will throughout the rest of this article be referred to with growth directions and growth times. Furthermore, the control surface N27Days of Sample 2, located $750 \mu \mathrm{m}$ away from the N-side of the seed crystal was in all measurements found to be practically identical to the corresponding surface of Sample 1, and it will therefore not be included in the following discussion. The wing region lengths are determined by the distance from the wing region edge to the crossing of the polished $(000 \pm 1)$ surfaces with $(000 \pm 1) /\{11 \overline{2} 2\}$ growth interfaces, denoted with solid lines in figure 2 (b). As an example, the wing region of surface N14Days is indicated with a horizontal arrow in figure 2 (b), the other wing regions are left unmarked for better intelligibility. The wing region lengths estimated from the sample geometry were approximately $830 \mu \mathrm{m}, 1060 \mu \mathrm{m}$ and $90 \mu \mathrm{m}$ for surfaces Ga15Days, N14Days and N27Days, respectively.

\subsection{Characterization}

The sample surfaces were characterized by SIMS, HRXRD, FTIR reflectivity, and low-temperature PL. SIMS was used to measure the oxygen, silicon and carbon impurity concentrations from the wing and seed regions of all surfaces. The measurements were taken from a square spot with dimensions of $150 \mu \mathrm{m} \times 150 \mu \mathrm{m}$.

To study the electrical effects of impurities, FTIR reflectivity line scans were measured from the wing region edge to sample middle edge, perpendicular to the wing/seed interface, as shown in figure 1. The spectra were recorded with a Bruker IFS66V spectrometer equipped with a Globar ${ }^{\text {TM }}$ light source and a liquid nitrogen-cooled mercury cadmium telluride detector, between spectral wavenumbers 600 and $40001 / \mathrm{cm}$, using both a spot diameter and a step size of $100 \mu \mathrm{m}$. The FTIR reflection curves were used to estimate the free-carrier concentration based on the wellknown Drude model [18, 19]. In the Drude model, the free-carrier density and drift mobility determine the shape of the FTIR reflection curve. In this work we used a Monte Carlo scheme to find the best fit to measurement data, providing unbiased estimates of the free carrier density.

Position dependent low temperature PL spectra were measured along the same lines as the FTIR spectra with a Horiba HR800 UV bench top system equipped with a monochromator with a 600 lines/mm grating and a CCDdetector. The individual spectra were recorded with both spot diameter and step size of $150 \mu \mathrm{m}$, and a temperature of approximately $4 \mathrm{~K}$, using a HeCd pump laser with a wavelength of $325 \mathrm{~nm}$.

The lattice constants $a$ and $c$ were measured with HR$\mathrm{XRD}$, using an x-ray mirror, a $4 \times$ Ge220 monochromator, an analyzer crystal and $\mathrm{Cu}-\mathrm{K}_{\alpha 1}$ radiation produced with $40 \mathrm{kV}$ acceleration voltage and $40 \mathrm{~mA}$ tube current. The wing and seed regions from both the Ga- and the N-faces were measured separately by restricting the beam size on the sample surface to $2 \times 1 \mathrm{~mm}^{2}$. This was accomplished by placing a copper foil with a routed hole on the sample surface. The method for determining the lattice constants was similar to the method described in Ref. [20]. The average lattice constant value $c$ was determined from four symmetric (0004) and four symmetric (0006) scans. The sample was offset $90^{\circ}$ in-plane between each scan and refraction correction was applied to obtain the corrected average value of $c$. This value was used in conjunction with

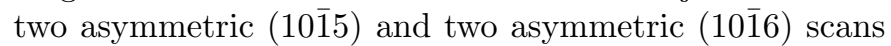
to determine the average value of $a$. The sample was offset $120^{\circ}$ in-plane between the asymmetric scans, and refraction corrections were applied. The errors in $a$ and $c$ were estimated by using the total differential with the standard deviations.

\section{Results \& discussion}

\section{1. $S I M S$}

The SIMS results for oxygen, silicon and carbon concentrations in the seed and wing regions of all studied 
surfaces are shown in table 1. The oxygen concentration varies between low $10^{19} \mathrm{~cm}^{-3}$ and mid $10^{20} \mathrm{~cm}^{-3}$, while the $\mathrm{Si}$ and $\mathrm{C}$ concentrations are essentially constant at low $10^{18} \mathrm{~cm}^{-3}$ and high $10^{17} \mathrm{~cm}^{-3}$, respectively. Since oxygen is a donor impurity and clearly the most abundant measured impurity, the sample is expected to be strongly ndoped. This would be consistent with Hall measurements performed on crystals grown in the same system and under comparable conditions 14 .

\section{[Table 1 about here.]}

The largest difference in impurity concentrations are found between the seed and the wing regions. The wing region of surface N14Days has roughly an order of magnitude higher concentration of oxygen than its seed region. The surface Ga15Days behaves similarly, the oxygen concentration in the wing region is twice that of its seed region. The wing area of surface N27Days is too small for reliable SIMS measurement, it was earlier estimated to be only $90 \mu \mathrm{m}$ in length. The SIMS results indicate that not only is oxygen incorporated in the wing regions more efficiently, but its incorporation efficiency differs also between both the wing and seed regions on the Ga- and N-sides of the crystal. The seed region of surface Ga15Days has a lower oxygen concentration than the seed regions of both N-polar surfaces N14Days and N27Days. This indicates, that regardless of a possibly decreasing oxygen concentration during growth, N-polar growth incorporates oxygen more efficiently than Ga-polar. The differences observed in wing regions will be discussed later in more detail.

The oxygen concentration of surface N14Days is only slightly higher than that of surface N27Days, suggesting that if the ambient impurity concentration reduces over time, its impact during the growth of $750 \mu \mathrm{m} \mathrm{GaN} \mathrm{is}$ rather small. The time-invariance of carbon, silicon, and to a large degree also oxygen, suggests the vast majority of the measured impurities originate from the source material and is released at a constant rate into the growth solution. Thus the differences in impurity concentration shown in table 1 originate mainly from differences in impurity incorporation efficiency of the wing and seed growth zones, not from variations during growth of the boule.

In light of the discussion in the previous paragraph, oxygen appears to incorporate in descending preferential order in the N-side wing, N-polar seed, Ga-side wing and Ga-polar seed regions. It is well known, that N-polar growth incorporates oxygen more efficiently than Ga-polar growth [21, 22, 23, but fewer reports exist on oxygen incorporation in other orientations. Especially interesting is that the wing regions of surfaces Ga15Days and N14Days have so dissimilar oxygen concentrations. A possible explanation is that growth occurred in several orientations, of which $\{11 \overline{2} 0\},\{10 \overline{1} 0\},\{10 \overline{1} 1\}$ and $\{11 \overline{2} 2\}$ are the most likely alternatives. Cruz et al. published a study on how impurities are incorporated in different orientations during metal organic chemical vapour deposition (MOCVD)
24]. They argued that the oxygen incorporation efficiency strongly depends on the surface density of nitrogen, resulting in high incorporation efficiency on N-rich planes, such as $\{11 \overline{2} 2\},\{10 \overline{1} 1\}$ and $\{000 \overline{1}\}$, and low incorporation efficiency on N-poor planes, e.g., $\{0001\}$ and $\{11 \overline{2} 0\}$. Despite their different growth method and much lower oxygen concentrations, the incorporation mechanism should not depend significantly on the growth method, and their results regarding oxygen incorporation should be qualitatively similar. This hypothesis is based on the similarity of the growth environments, that is the presence of $\mathrm{NH}_{3}$, $\mathrm{N}_{2}$ and $\mathrm{H}_{2}$ in both ammonothermal growth and MOCVD. Figure 3 shows a plot of the oxygen concentrations observed in this study and oxygen concentrations adapted from the publication of Cruz et al.. The filled symbols represent values measured in this study, the empty symbols represent results from the MOCVD growth in Ref [24]. For clarity, the names of surfaces Ga15Days and N14Days are shortened to $\mathrm{Ga}$ and $\mathrm{N}$ in figure 3 . The two datasets in figure 3 have been laterally aligned using the known orientations, that is Ga seed $=(0001)$ and $\mathrm{N}$ seed $=(000 \overline{1})$. The two trends show a remarkable similarity, suggesting that the N14Days wing area was grown in the [11 22$]$-direction, consistent with the earlier observation of the $(11 \overline{2} 2)$ face in the wing region in figure 1 . The similar relative concentrations of the Ga15Days wing region and the MOCVD grown (1010) GaN raises the question if a part of the Ga15Days wing region was grown in the [10̄10]-direction. This is a possible explanation, since a-planes have been observed to facet into m-planes in previous growths [12, but a definitive conclusion cannot be drawn from the SIMS-data. It is clear from this result that the wing regions contain areas of contrasting impurity levels and that the wing regions can therefore not be assumed to be homogeneous. Since the SIMS measurement area is only $150 \mu \mathrm{m} \times 150 \mu \mathrm{m}$, it is still unclear how large the inhomogeneous areas are, and whether there are general differences between the Ga- and $\mathrm{N}$-sides. It should additionally be noted, that an increase in growth rate often results in an enhanced impurity trapping, and consequently in more efficient impurity incorporation in the growing crystal. However, as the differences in [O] follow the observed growth sectors, impurity trapping due to variations in growth rate does not seem to be the dominating impurity incorporation mechanism.

\section{[Figure 3 about here.]}

\section{2. $X R D$}

The observed oxygen concentration differences between different growth regions are enough to cause significant variation in the lattice constants and are thus expected to induce stress in the crystal. The lattice constants measured by HR-XRD from the wing and seed regions of different surfaces are shown in figure 4. The filled symbols represent the seed regions, while the empty symbols represent the corresponding wing regions. The strikingly large 
error bars in figure 4 are a result of significant inhomogeneity, seen as multiple diffraction peaks in several of the XRD-scans.

[Figure 4 about here.]

A comparison of figure 4 and table 1 indicates the lattice constants are heavily influenced by the changes in the oxygen concentration. The smallest lattice constant $c$, marked with a filled square in figure 4. was measured from the seed region of surface Ga15Days, and corresponds to the lowest oxygen concentration measured by SIMS. The other, N-polar grown seed regions have slightly larger and within measurement accuracy equal lattice constants, as was expected from the SIMS results. In agreement with the growth model presented in figure 2, according to which the wing area on the $\mathrm{N}$-side of the crystal is negligibly small, the lattice constants of the surface N27Days showed no spatial variation, and therefore only the results of the seed region are shown in figure 4. A more dramatic change in the lattice constants is observed for the wing regions of surfaces Ga15Days and N14Days. The lattice constant $c$ of Ga15Days changes from its seed region value of 5.1852 $\AA$ to $5.1858 \AA$ in the wing region. The change in the lattice constant of surface N14Days is even larger and changes from $5.1854 \AA$ to $5.1861 \AA$ between the seed and wing regions, respectively. However, due to the large degree of inhomogeneity, too much weight should not be put on the exact values of the lattice constants. They are however in good agreement with previously reported lattice constants of ammonothermal GaN with similar oxygen concentrations [16, 25].

Despite the uncertainties in the exact values of the lattice constants, it can be concluded from figure 4 that the Ga15Days wing region has a larger lattice than the seed regions. This contrasts the SIMS measurements, according to which the Ga15Days wing region has a lower oxygen concentration than the N-polar seed regions. This result, and the large error bars, further supports the previously presented hypothesis that the wing region is inhomogeneous on a relatively small scale. The inhomogeneity of the wing regions will be discussed more in the following section.

The changes in the lattice constant values correlate qualitatively with the variation in the oxygen concentration measured by SIMS, but the effect of high oxygen concentrations on the lattice parameters may be quantified based on calculations by Van de Walle 26]. According to Van de Walle, the lattice expansion or contraction may be estimated with the help of the deformation potential and the size-effect of the impurity atom. Based on the measured lattice parameters and Ref [26, an oxygen concentration of $6.3 \times 10^{19} \mathrm{~cm}^{-3}$ was calculated for the N-polar seed regions, which is in excellent agreement with the measured oxygen concentrations $5.2-7.5 \times 10^{19} \mathrm{~cm}^{-3}$. In the calculation, the $\mathrm{N}$-polar seed regions were assumed to be fully relaxed, and therefore the lattice expansion could be assumed hydrostatic. The actual calculation was carried out for the lattice parameter $c$, since $a$ could not be determined directly, and the error in $c$ therefore adds to the error in $a$. Similar calculations yield oxygen concentrations of $4.7 \times 10^{19} \mathrm{~cm}^{-3}$ for the Ga15Days seed region, $9.3 \times 10^{19}$ $\mathrm{cm}^{-3}$ for the Ga15Days wing region and $1.2 \times 10^{20} \mathrm{~cm}^{-3}$ for the N14Days wing region. These values are in satisfactory agreement with the SIMS results in table 1, in particular considering the large error bars associated with the lattice constants.

\subsection{Free-carrier concentration}

Figure 5 shows the free carrier concentrations $n$ obtained by fitting the Drude model to the FTIR measurements. The free carrier concentrations are shown as a function of distance from the wing/seed interface. The positions of the steps in the free carrier concentration profiles were taken as the positions of the wing/seed interfaces. The net donor impurity concentration, excluding contributions from native defects, e.g. Ga-vacancies, $N_{D}-N_{A}=$ $[\mathrm{O}]+[\mathrm{Si}]-[\mathrm{C}]$ obtained from SIMS is also shown in figure 5 for each surface and growth region. The free carrier concentration profiles in figure 5 have generally similar shapes to their corresponding net donor concentration profiles; the free carrier concentrations are markedly higher in the wing regions than in the seed regions. This is in agreement with the darkening of the wing regions seen in figure 1 . most likely related to the increased free carrier concentration and not an increased impurity uptake [14. Surfaces Ga15Days and N14Days have well defined wing regions, whereas the surface N27Days displays a small wing region consisting of only two measurement points. The spectrum of the first point (not shown here) is a mixture of low and high free carrier concentration contribution, whereas the second spectrum is caused solely by a higher free carrier concentration. This indicates the length of the wing region of surface N27Days is approximately $100 \mu \mathrm{m}$, and that the lengths of the wing regions in figure 5 are in good agreement with the values estimated in Section 2 Because the length of the wing region depends on the growth time, and the free carrier concentrations in the wing regions are roughly constant, the oxygen concentration is essentially time-invariant. This result further supports our earlier conclusion that the majority of the measured impurities have their origins in the source material and not in the ambient.

\section{[Figure 5 about here.]}

The net donor concentrations in the seed regions of all surfaces are to a varying degree higher than the free carrier concentrations, indicating the donor impurities are compensated by something other than $\mathrm{C}$. The Ga vacancy, and/or its complexes is the most viable option, since it has frequently been observed in ammonothermal GaN [14, 27, 28, and other acceptor impurity atoms have typically 
far lower concentrations. As seen in figure 5, the difference between the net donor concentration and the free carrier concentration varies with growth direction and region. Since the $\mathrm{V}_{\mathrm{Ga}}$ concentration has been reported to follow the oxygen concentration [29], the difference between the net donor concentrations and free carrier concentrations should increase with increasing oxygen concentration, if Ga-vacancies and/or its complexes were to form the dominant compensation mechanism. As indicated in figure 5, this is indeed the case for all regions, but the wing region of surface Ga15Days. An acceptor type $\mathrm{V}_{\mathrm{Ga}}-\mathrm{H}_{\mathrm{x}}$ defect complex concentration of $\approx 3 \times 10^{18} \mathrm{~cm}^{-3}$ was recently observed in ammonothermal GaN, and suggested to largely explain the difference between net donor and free carrier concentrations of $\approx 1 \times 10^{19} \mathrm{~cm}^{-3}$ and $\approx 2 \times 10^{18} \mathrm{~cm}^{-3}$, respectively 30 . Although the oxygen concentrations in this study are higher, the same major compensation mechanism seems viable. Interestingly, the wing region of surface Ga15Days shows no sign of compensation. As discussed earlier though, the wing region seems to be very inhomogeneous even on a small scale, and the equal free carrier and net donor concentrations are therefore likely to correspond to two different regions. To check the homogeneity of the wing region, several FTIR linescans were measured within the wing region, perpendicular to the linescan shown in figure 11. Quite a few of the spectra (not shown here) appear to be hybrids of FTIR spectra from high free carrier concentration material and FTIR spectra from low free carrier concentration material, indicating the result in figure 5 is caused by inhomogeneity. Additional work on identifying the growth surfaces and evolution of Ga-wing during growth of the boule would provide greater clarity on the origin of the inhomogeneity, in particular if they originate from a highly faceted growth of mixed planes (m-plane, a-plane, $\{11 \overline{2} 2\}$ planes). It is not unlikely that this could occur due to the unpolished nature of the non-polar side walls of the seed from which the Ga-face wing originated.

\subsection{Photoluminescence}

Figure 6 presents examples of PL spectra from the seed and the wing regions of surfaces Ga15Days and N14Days. The spectra were extracted from the line scans. The surface N27Days will be omitted from the following discussion, because its wing region is too small for reliable measurements. The optics of the PL-setup introduces oscillatory artefacts from around $440 \mathrm{~nm}$ onwards, and these were successfully filtered prior to further analysis, except for the remaining bump centred around $460 \mathrm{~nm}$. An example of an unfiltered scan is also presented in figure 6. Despite the oscillations and remaining artefacts, relative comparisons can be made within individual samples. The spectra are characterized by the near band edge (NBE) luminescence peak at $357 \mathrm{~nm}$, the donoracceptor pair (DAP) at $376 \mathrm{~nm}$ and broad blue luminescence (BL) and yellow luminescence (YL) bands at $420 \mathrm{~nm}$ and $560 \mathrm{~nm}$, respectively. The position of the NBE emission matches well with reported positions of donor-bound excitonic emission with similar free carrier concentrations 31, but individual $\mathrm{O}$ - and Si-bound exciton peaks are spectrally too close to be resolved 32]. The NBE may be attributed to mainly O-bound excitons, based on the high oxygen concentration. The BL and YL bands have previously been assigned to $\mathrm{Zn}, \mathrm{C}, \mathrm{Mg}$ and Ga-vacancies and its complexes [33, 34, 35, 36. The Ga-vacancy and its complexes are a plausible impurity luminescence source, since the C-concentration was measured to be fairly low, previous studies have showed the $\mathrm{Mg}$-concentration to be also quite low 13 and $\mathrm{Zn}$ is not a common impurity in ammonothermal GaN. Although the BL band dominates each spectrum, it is clear that the optical properties differ between different surfaces and growth regions. The spatial variation of the integrated intensities and peak positions will therefore be discussed in the following paragraphs.

\section{[Figure 6 about here.]}

As seen in figure 6, the NBE peak blueshifts and broadens and the DAP peak disappears in the wing regions. The positions of the NBE emission peaks shifted from 358.0 $\mathrm{nm}$ to $352.6 \mathrm{~nm}$ for surface Ga15Days and from 357.3 $\mathrm{nm}$ to $352.0 \mathrm{~nm}$ for surface N14Days. Such observations are typically caused by the Burstein-Moss effect, in which a very high doping level shifts the Fermi level up inside the conduction band, resulting in an increased band gap [37. These results are in line with the previously discussed higher free carrier concentration in the wing regions.

The integrated intensities of the NBE, BL and YL bands, and the free carrier concentrations are shown in figure 7 as functions of distance. To better visualize the change between the seed and wing regions, the intensities were normalized to equal medians in the seed regions. Both surfaces show a reduction in the BL and YL intensities in the wing regions, despite an increase in oxygen and free carrier concentrations and a near constant carbon concentration. The NBE luminescence of surface Ga15Days increases significantly in the wing region, while that of surface N14Days decreases slightly. Figure 7 suggests that a large increase in free carrier concentration causes also a large increase in the NBE luminescence. In contrast, a small increase in free carrier concentration on surface N14Days is accompanied with a slightly decreased NBE luminescence.

\section{[Figure 7 about here.]}

\section{Conclusions}

In this article we reported on the distribution of impurities in different growth zones and at different growth times within a single ammonothermally grown GaN boule. The impurity concentrations were measured using SIMS, and their effects on the lattice size were measured by HR-XRD. 
The electrical properties were studied by measuring the spatially resolved free carrier concentration and the optical properties were determined by low-temperature PL. The results reveal that oxygen is the most abundant impurity species, and its incorporation efficiency varies dramatically between seed and wing growth zones. We further observe large differences in oxygen incorporation efficiency within wing regions, and we attribute the varying oxygen incorporation predominantly to growth in different directions. The stark difference in impurity uptake between wing and seed regions causes a significant expansion of the lattice, resulting in non-negligible strain within the boule. The impurity concentrations were not found to vary greatly with growth time, suggesting they are sourced from the polycrystalline GaN source material. The impurity concentration profiles result in similarly, albeit compensated, free carrier concentration profiles. The varying impurity incorporation was found to clearly affect the PL intensity.

\section{Acknowledgments}

The research was in part supported by the Academy of Finland and Aalto Energy Efficiency Programme. Additional support is acknowledged from the Solid State Lighting and Energy Electronics Center at University of California, Santa Barbara and the MRL Central Facilities, which are supported by the MRSEC Program of the NSF under Award no. DMR 1121053; a member of the NSF- funded Materials Research Facilities Network (www.mrfn.org). Great gratitude is extended to Takatori Corporation for their generous donation of the wire saw used to cut the GaN samples. Kivisaari wants to express his gratitude to the Jenny and Antti Wihuri Foundation, the Nokia Foundation, and the Finnish Cultural Foundation for supporting his research. Sintonen wishes to thank Svenska Tekniska Vetenskapsakademien i Finland and the Jenny and Antti Wihuri Foundation for their generous financial support.

\section{References}

[1] S. Pimputkar, J. S. Speck, S. P. DenBaars, S. Nakamura, Prospects for LED lighting, Nature Photon. 3 (4) (2009) 180182. doi:10.1038/nphoton.2009.32

[2] S. Nakamura, M. R. Krames, History of Gallium-Nitride-Based Light-Emitting Diodes for Illumination, Proc. IEEE 101 (10) (2013) 2211-2220. doi:10.1109/JPROC.2013.2274929

[3] C. A. Hurni, A. David, M. J. Cich, R. I. Aldaz, B. Ellis, K. Huang, A. Tyagi, R. a. Delille, M. D. Craven, F. M. Steranka, M. R. Krames, C. a. Hurni, A. David, M. J. Cich, R. I. Aldaz, B. Ellis, K. Huang, A. Tyagi, R. A. Delille, M. D. Craven, F. M. Steranka, Bulk GaN flip-chip violet light-emitting diodes with optimized efficiency for high-power operation, Appl. Phys. Lett. 031101. doi:10.1063/1.4905873

[4] T. D. Heidel, B. A. Hamilton, Power Devices on Bulk Gallium Nitride Substrates : An Overview of ARPA-E's SWITCHES Program, IEEE International Electron Devices Meeting (2014) 44-47doi:10.1109/IEDM.2014.7046969

[5] D. Wang, A. Pierre, M. G. Kibria, K. Cui, X. Han, K. H. Bevan, H. Guo, S. Paradis, A.-R. Hakima, Z. Mi, Wafer-level photocatalytic water splitting on gan nanowire arrays grown by molecular beam epitaxy, Nano Lett. 11 (6) (2011) 23532357, pMID: 21568321. arXiv:http://dx.doi.org/10.1021/ nl2006802 doi:10.1021/nl2006802

[6] S. Li, A. Waag, Gan based nanorods for solid state lighting, J. Appl. Phys. 111 (7). doi:http://dx.doi.org/10.1063/1. 3694674

[7] D. Bayerl, E. Kioupakis, Visible-wavelength polarized-light emission with small-diameter inn nanowires, Nano Lett. 14 (7) (2014) 3709-3714, pMID: 24527880. arXiv:http://dx.doi. org/10.1021/nl404414r doi:10.1021/nl404414r.

[8] L. Riuttanen, P. Kivisaari, O. Svensk, J. Oksanen, S. Suihkonen, Electrical injection to contactless near-surface ingan quantum well, Appl. Phys. Lett. 107 (5). doi:http://dx.doi.org/10. 1063/1.4928248

[9] T. Paskova, D. A. Hanser, K. R. Evans, T. Paskova, D. A. Hanser, K. R. Evans, G. Substrates, I.-n. Devices, GaN Substrates for III-Nitride Devices, Proc. IEEE 98 (7) (2010) 13241338. doi:10.1109/JPROC.2009.2030699

[10] D. Bliss, B. Wang, M. Suscavage, R. Lancto, S. Swider, W. Eikenberry, C. Lynch, Ammonothermal gan: Morphology and properties, J. Cryst. Growth 312 (8) (2010) 1069 - 1073, the 17th American Conference on Crystal Growth and Epitaxy/The 14th \{US\} Biennial Workshop on Organometallic Vapor Phase Epitaxy/The 6th International Workshop on Modeling in Crystal Growth. doi:http://dx.doi.org/10.1016/j. jcrysgro.2009.12.007

[11] R. Dwiliński, R. Doradziński, J. Garczyński, L. Sierzputowski, R. Kucharski, M. Zajac, M. Rudziński, R. Kudrawiec, W. Strupiński, J. Misiewicz, Ammonothermal GaN substrates: Growth accomplishments and applications, Phys. Stat. Solidi (A) 208 (7) (2011) 1489-1493. doi:10.1002/pssa. 201001196

[12] S. Pimputkar, S. Kawabata, J. Speck, S. Nakamura, Surface morphology study of basic ammonothermal GaN grown on nonpolar GaN seed crystals of varying surface orientations from m-plane to a-plane, J. Cryst. Growth 368 (2013) 67-71. doi: 10.1016/j.jcrysgro.2013.01.022

[13] S. Pimputkar, S. Kawabata, J. Speck, S. Nakamura, Improved growth rates and purity of basic ammonothermal GaN, J. Cryst. Growth 403 (2014) 7-17. doi:10.1016/j.jcrysgro.2014.06. 017

[14] S. Pimputkar, S. Suihkonen, M. Imade, Y. Mori, J. Speck, S. Nakamura, Free electron concentration dependent subbandgap optical absorption characterization of bulk GaN crystals, J. Cryst. Growth 432 (2015) 49-53. doi:10.1016/j. jcrysgro.2015.09.016

[15] S. Sintonen, M. Rudziński, S. Suihkonen, H. Jussila, M. Knetzger, E. Meissner, A. Danilewsky, T. O. Tuomi, H. Lipsanen, Synchrotron radiation $\mathrm{x}$-ray topography and defect selective etching analysis of threading dislocations in GaN, J. Appl. Phys. 116 (8) (2014) 0-9. doi:10.1063/1.4893901

[16] S. Sintonen, S. Suihkonen, H. Jussila, A. Danilewsky, R. Stankiewicz, T. O. Tuomi, H. Lipsanen, Large-area analysis of dislocations in ammonothermal GaN by synchrotron radiation X-ray topography, Appl. Phys. Express 7 (9) (2014) 10-13. doi:10.7567/APEX.7.091003

[17] M. Saito, D. S. Kamber, T. J. Baker, K. Fujito, S. P. DenBaars, J. S. Speck, S. Nakamura, Plane dependent growth of GaN in supercritical basic ammonia, Appl. Phys. Express 1 (12) (2008) 1211031-1211033. doi:10.1143/APEX.1.121103

[18] A. Barker, M. Ilegems, Infrared Lattice Vibrations and FreeElectron Dispersion in GaN, Phys.Rev. B 7 (2) (1973) 743-750. doi:10.1103/PhysRevB.7.743

[19] B. Birkmann, C. Salcianu, E. Meissner, S. Hussy, J. Friedrich, G. Müller, Characterisation of the electrical properties of solution-grown GaN crystals by reflectivity and Hall measurements, Phys. Stat. Solidi (C) 3 (3) (2006) 575-578. doi: 10.1002/pssc.200564119.

[20] V. Darakchieva, T. Paskova, P. P. Paskov, B. Monemar, N. Ashkenov, M. Schubert, Structural characteristics and lattice parameters of hydride vapor phase epitaxial GaN free- 
standing quasisubstrates, J. Appl. Phys. 97 (1) (2005) 013517. doi:10.1063/1.1823024

[21] M. Sumiya, K. Yoshimura, K. Ohtsuka, S. Fuke, Dependence of impurity incorporation on the polar direction of GaN film growth, Appl. Phys. Lett. 76 (15) (2000) 2098-2100. doi:doi: 10.1063/1.126267

[22] E. Frayssinet, W. Knap, S. Krukowski, P. Perlin, P. Wisniewski, T. Suski, I. Grzegory, S. Porowski, Evidence of free carrier concentration gradient along the $\mathrm{c}$-axis for undoped GaN single crystals, J. Cryst. Growth 230 (2001) 442-447. doi: 10.1016/S0022-0248(01)01294-5.

[23] N. A. Fichtenbaum, T. E. Mates, S. Keller, S. P. DenBaars, U. K. Mishra, Impurity incorporation in heteroepitaxial N-face and Ga-face GaN films grown by metalorganic chemical vapor deposition, J. Cryst. Growth 310 (6) (2008) 1124-1131. doi: $10.1016 / j \cdot j$ crysgro.2007.12.051

[24] S. C. Cruz, S. Keller, T. E. Mates, U. K. Mishra, S. P. DenBaars, Crystallographic orientation dependence of dopant and impurity incorporation in GaN films grown by metalorganic chemical vapor deposition, J. Cryst. Growth 311 (15) (2009) 3817-3823. doi:10.1016/j.jcrysgro.2009.02.051

[25] D. Gogova, P. P. Petrov, M. Buegler, M. R. Wagner, C. Nenstiel, G. Callsen, M. Schmidbauer, R. Kucharski, M. Zajac, R. Dwilinski, M. R. Phillips, A. Hoffmann, R. Fornari, Structural and optical investigation of non-polar (1-100) GaN grown by the ammonothermal method, J. Appl. Phys. 113 (20) (2013) 203513. doi:10.1063/1.4807581

[26] C. Van de Walle, Effects of impurities on the lattice parameters of GaN, Phys. Rev.B 68 (16) (2003) 165209. doi:10.1103/ PhysRevB.68.165209

[27] F. Tuomisto, J.-M. Mäki, M. Zając, Vacancy defects in bulk ammonothermal GaN crystals, J. Cryst. Growth 312 (18) (2010) 2620-2623. doi:10.1016/j.jcrysgro.2010.04.023

[28] F. Tuomisto, T. Kuittinen, M. Zajac, R. Doradziński, D. Wasik, Vacancy-hydrogen complexes in ammonothermal GaN, J. Cryst. Growth 403 (2014) 114-118. doi:10.1016/j.jcrysgro.2014. 06.005

[29] F. Tuomisto, K. Saarinen, B. Lucznik, I. Grzegory, H. Teisseyre, T. Suski, S. Porowski, P. R. Hageman, J. Likonen, Effect of growth polarity on vacancy defect and impurity incorporation in dislocation-free GaN, Appl. Phys. Lett. 86 (3) (2005) 1-3. doi:10.1063/1.1854745

[30] S. Suihkonen, S. Pimputkar, J. S. Speck, S. Nakamura, Infrared absorption of hydrogen-related defects in ammonothermal gan, Applied Physics Letters 108 (20). doi:http://dx.doi.org/10. 1063/1.4952388

URL http://scitation.aip.org/content/aip/journal/apl/ 108/20/10.1063/1.4952388

[31] H. Siegle, a. Hoffmann, L. Eckey, C. Thomsen, J. Christen, F. Bertram, D. Schmidt, D. Rudloff, K. Hiramatsu, Vertical strain and doping gradients in thick GaN layers, Appl. Phys. Lett. 71 (17) (1997) 2490-2492. doi:10.1063/1.120097.

[32] J. A. Freitas, B. N. Feigelson, T. J. Anderson, Efficient incorporation of $\mathrm{Mg}$ in solution grown GaN crystals, Appl. Phys. Express 6 (11). doi:10.7567/APEX.6.111001

[33] D. O. Demchenko, M. a. Reshchikov, Blue luminescence and Zn acceptor in GaN, Phys. Rev. B 88 (11) (2013) 1-6. doi: 10.1103/PhysRevB.88.115204

[34] M. a. Reshchikov, D. O. Demchenko, a. Usikov, H. Helava, Y. Makarov, Carbon defects as sources of the green and yellow luminescence bands in undoped GaN, Phys. Rev. B 235203 (2014) 1-16. doi:10.1103/PhysRevB.90.235203.

[35] J. L. Lyons, A. Alkauskas, A. Janotti, C. G. Van de Walle, First-principles theory of acceptors in nitride semiconductors, Phys. Stat. Solidi (B) 9. doi:10.1002/pssb. 201552062

[36] D. O. Demchenko, I. C. Diallo, M. a. Reshchikov, Yellow luminescence of gallium nitride generated by carbon defect complexes, Phys. Rev. Lett. 110 (8) (2013) 1-5. doi:10.1103/ PhysRevLett.110.087404

[37] P. Prystawko, M. Leszczynski, B. Beaumont, P. Gibart, E. Frayssinet, W. Knap, P. Wisniewski, M. Bockowski, T. Suski,
S. Porowski, Doping of homoepitaxial GaN layers, Phys. Stat. Solidi (B) 210 (2) (1998) 437-443. doi:10.1002/(SICI) 1521-3951(199812) 210:2<437: :AID-PSSB437>3.0.CO;2-L 

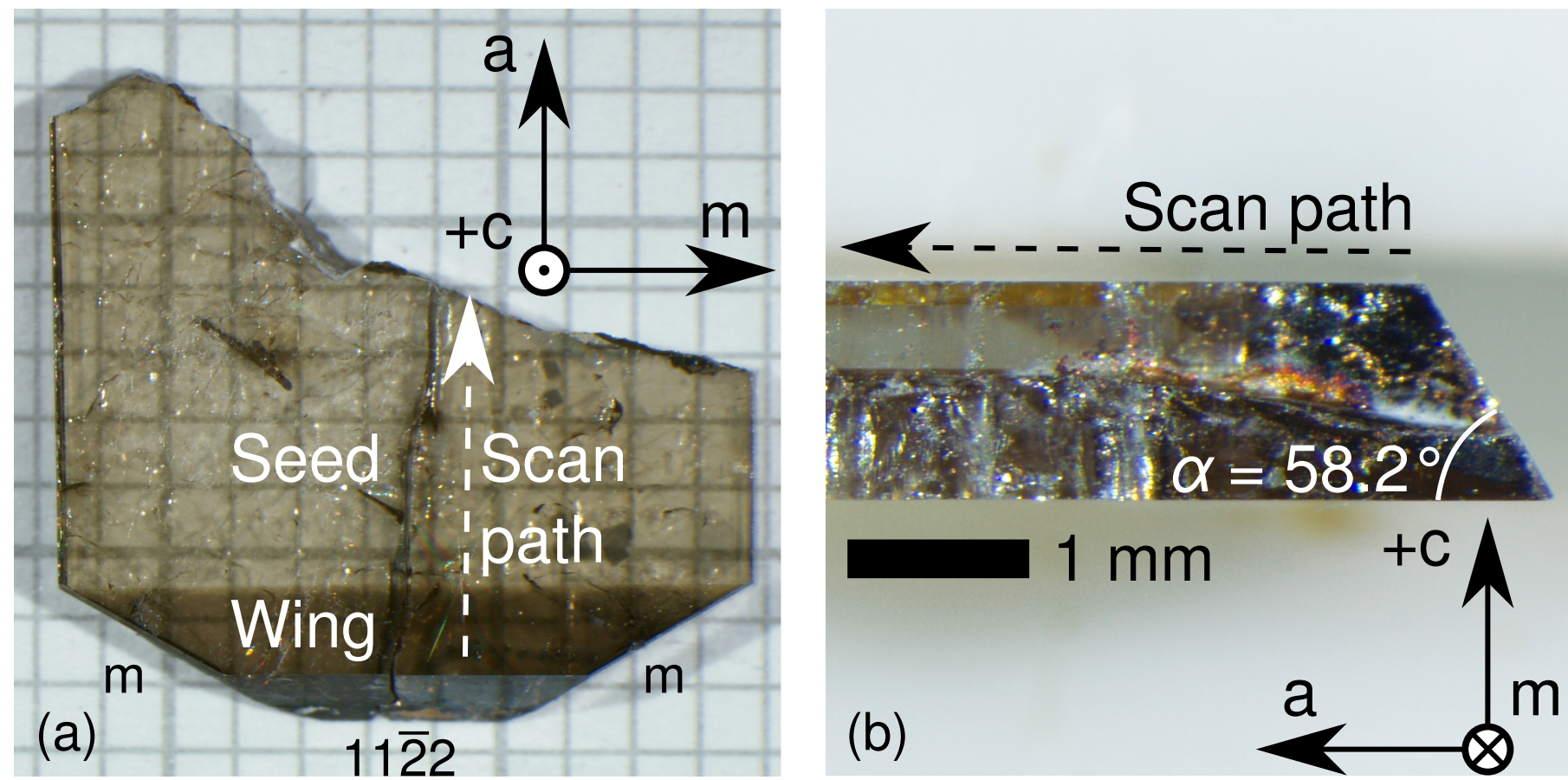

Figure 1: Reflected light images of Sample 1 Ga-surface (a) and m-plane cross-section (b) on millimeter paper after growth, thinning, and polishing. The dashed arrows show the paths along which the FTIR reflection and PL measurements are taken. 


\section{Ga-Polar}

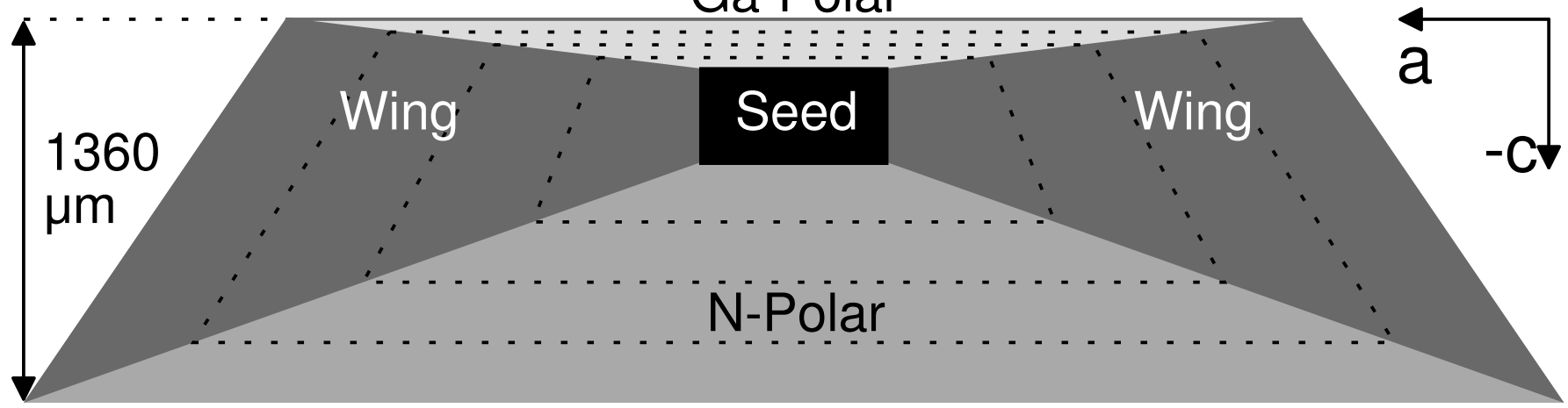

(a)

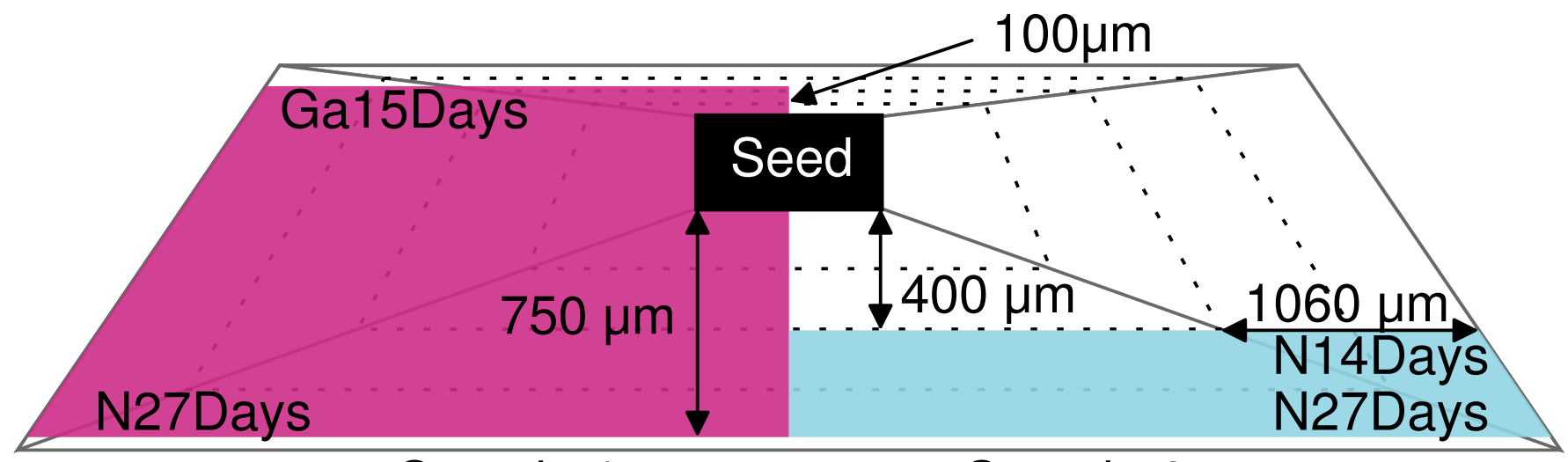

Sample 1

(b)

Figure 2: Schematic cross section illustrating (a) the evolution of the different growth regions and (b) the locations of the studied surfaces Ga15Days, N14Days and N27Days. 


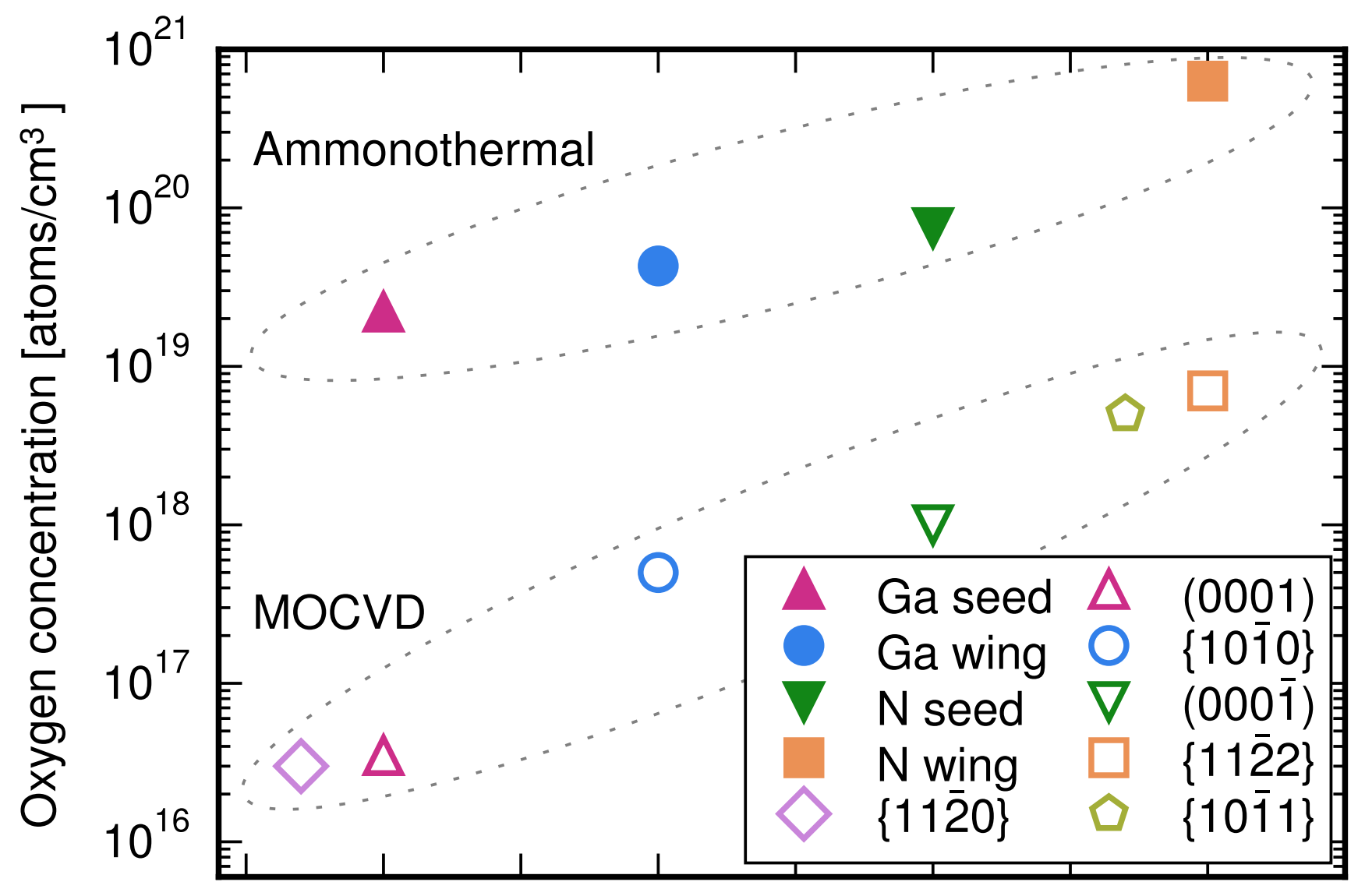

Surface/orientation

Figure 3: Oxygen concentrations in different growth regions and growth directions. Filled symbols represent oxygen concentrations observed in this work, while empty symbols were adapted from Ref [24]. 


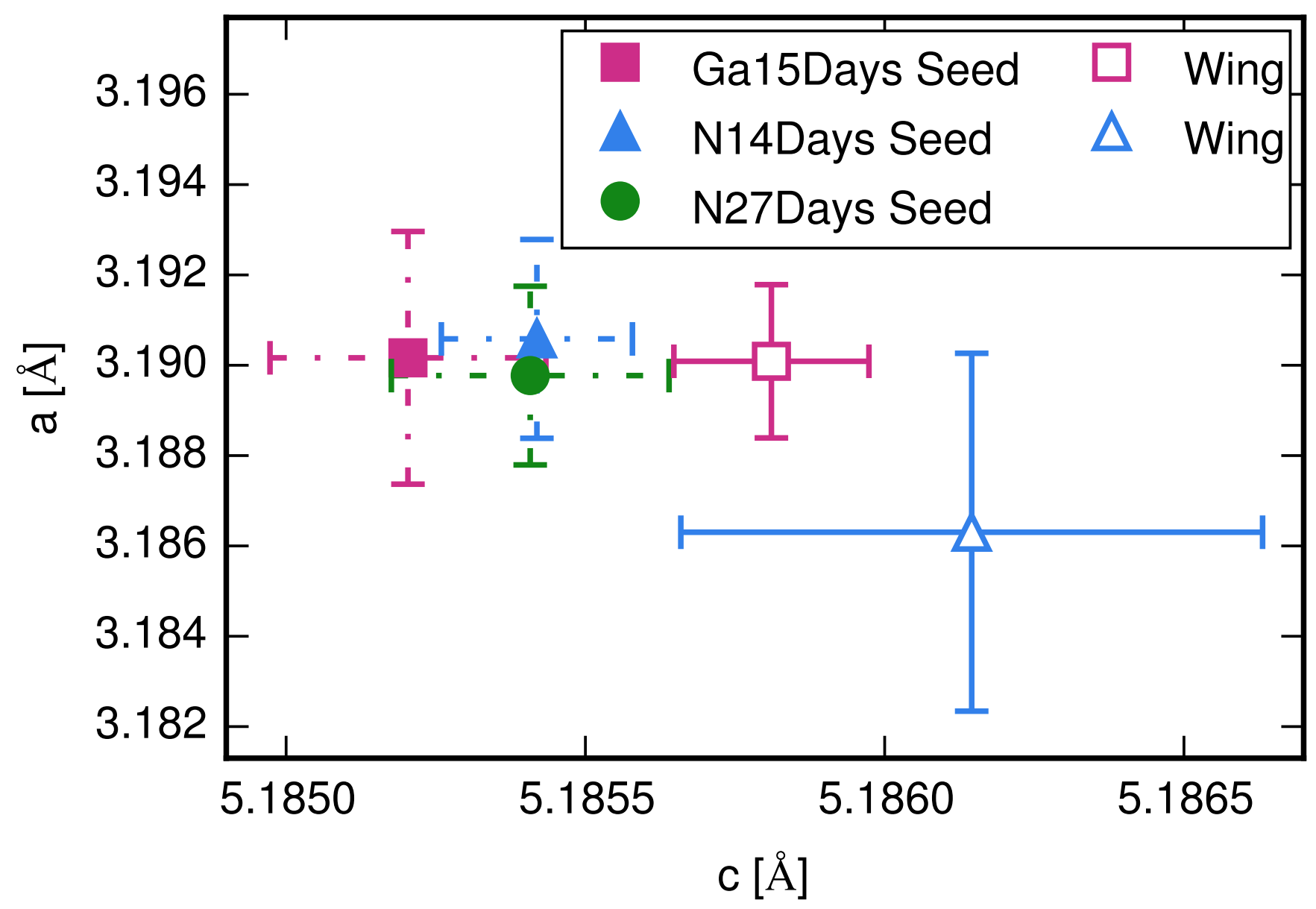

Figure 4: Lattice constants measured by HR-XRD from different regions and surfaces. Filled symbols represent seed regions, while empty symbols represent corresponding wing regions. 


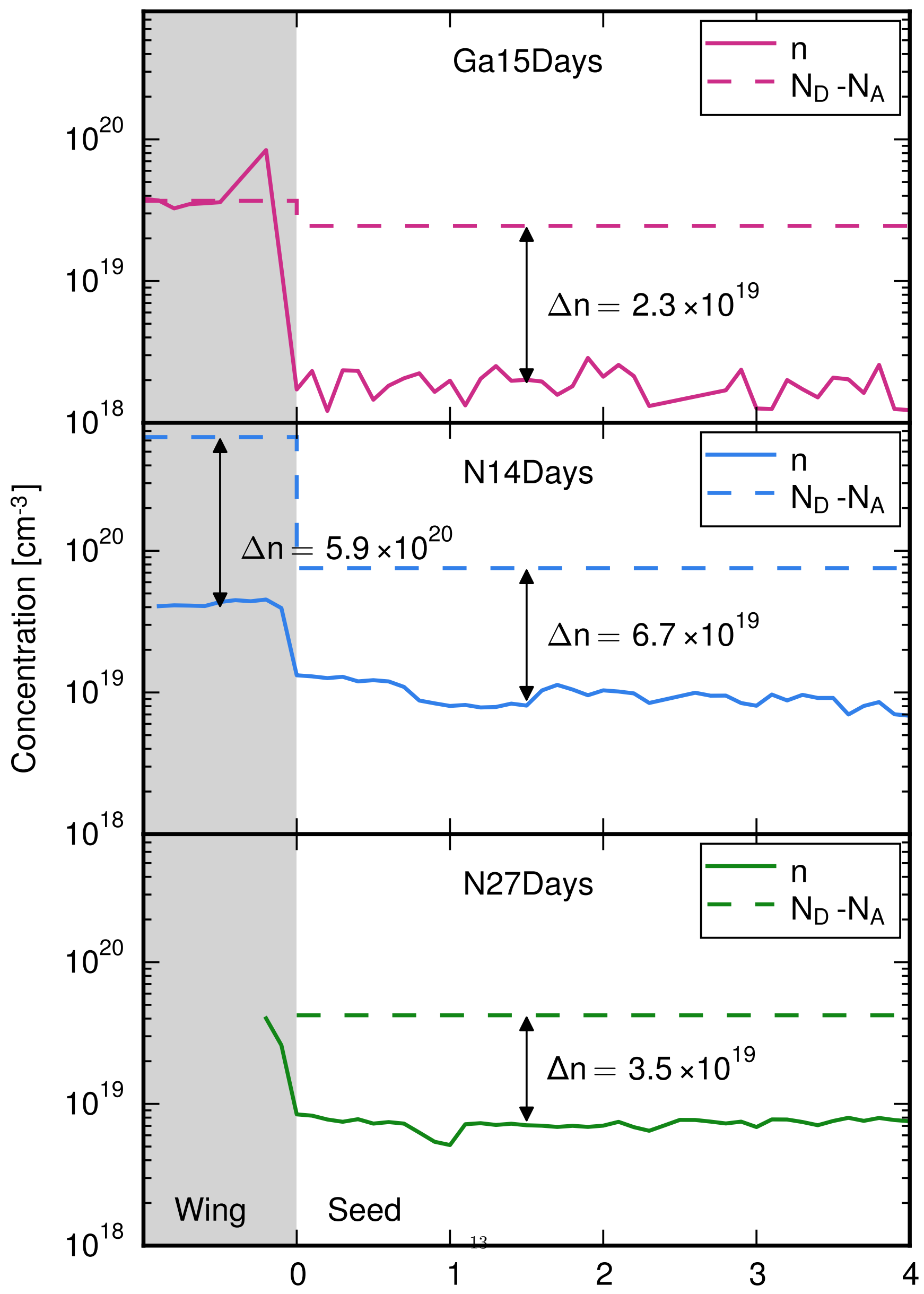

Distance from wing/seed interface [mm] 


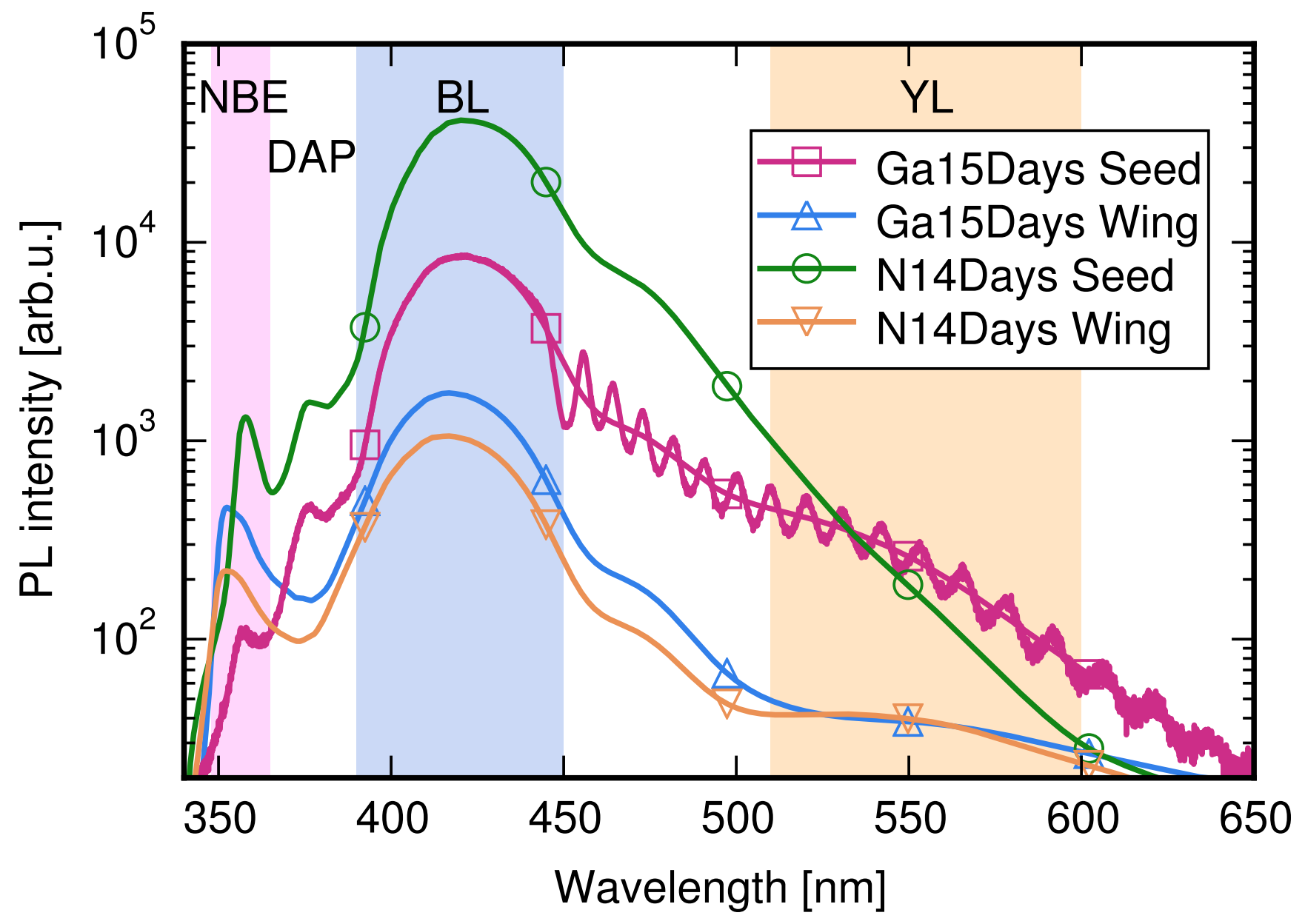

Figure 6: Examples of photoluminescence spectra recorded from the wing and seed regions of surfaces Ga15Days and N14Days. One unfiltered spectrum is also shown for reference. 


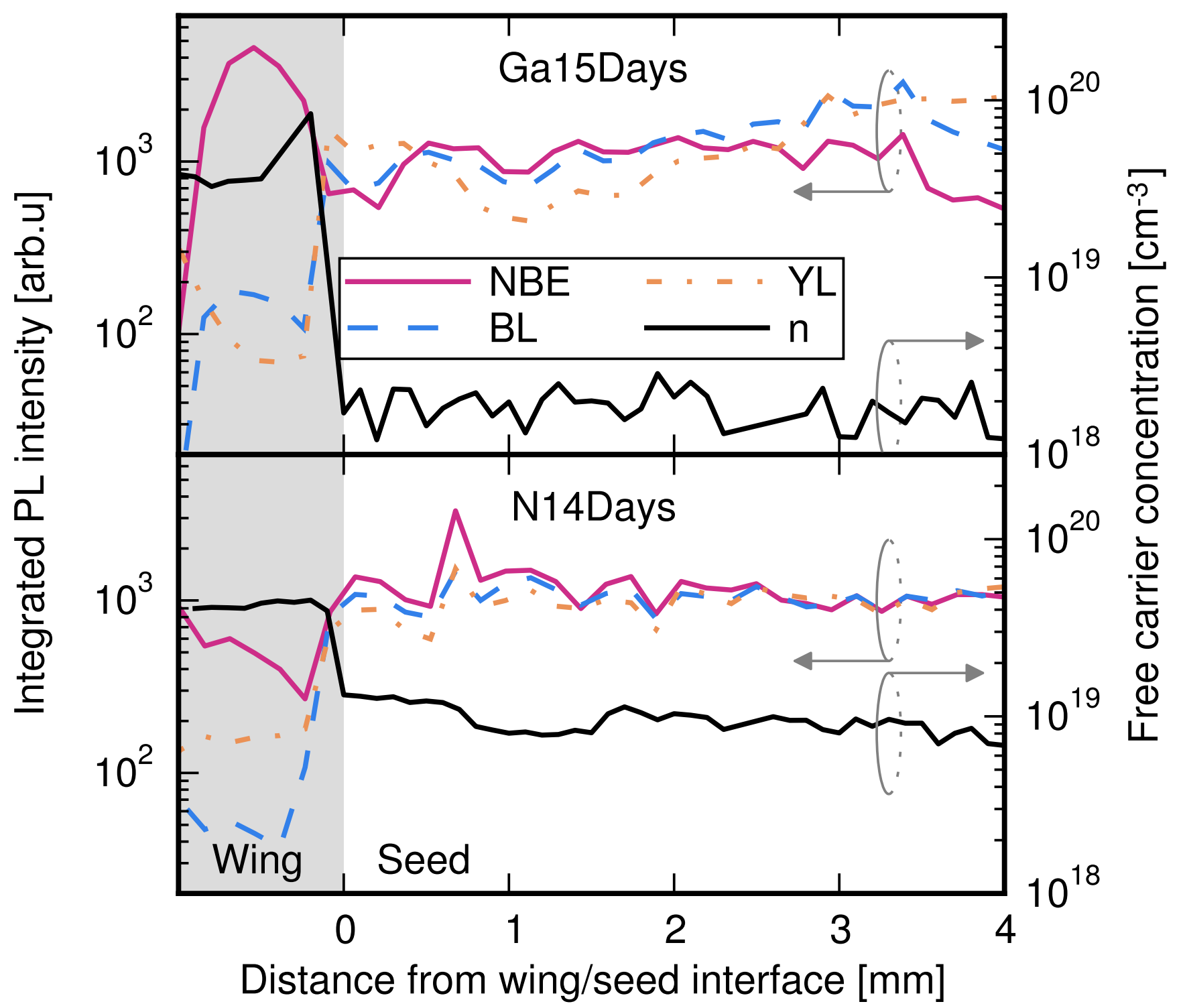

Figure 7: Relative integrated PL intensities and free carrier concentrations as a function of position for surfaces Ga15Days and N14Days 


\begin{tabular}{lccccccc}
\hline Surface & \multicolumn{3}{c}{ Seed $\left[10^{19} \mathrm{~cm}^{-3}\right]$} & \multicolumn{3}{c}{ Wing $\left[10^{19} \mathrm{~cm}^{-3}\right]$} \\
\cline { 2 - 4 } & {$[\mathrm{O}]$} & {$[\mathrm{Si}]$} & {$[\mathrm{C}]$} & & {$[\mathrm{O}]$} & {$[\mathrm{Si}]$} & {$[\mathrm{C}]$} \\
\hline Ga15Days & 2.2 & 0.4 & 0.2 & & 4.3 & 0.1 & 0.1 \\
N14Days & 7.5 & 0.1 & 0.1 & & 63.2 & 0.5 & 0.1 \\
N27Days & 5.2 & 0.1 & 0.1 & & - & - & - \\
\hline
\end{tabular}

Table 1: SIMS measurement results of oxygen, silicon and carbon concentrations in different growth regions. 